\title{
DISEASE ECOLOGY OF FREE-RANGING DOGS IN CENTRAL INDIA: IMPLICATIONS FOR WILDLIFE CONSERVATION
}

\author{
Aniruddha Belsare \\ Dr. Matthew Gompper, Dissertation Supervisor
}

\begin{abstract}
I obtained baseline demographic and epidemiologic data for village dog populations around a protected area in central India, with the objective of evaluating the risks dog populations present to sympatric wild carnivores. Demographic surveys indicated that dogs occurred at high densities in the study villages ( $>526$ dogs per $\mathrm{km}^{2}$ ). Epidemiological surveys documented a high prevalence of exposure to three pathogens of conservation concern, canine parvovirus (CPV), canine distemper virus (CDV), and canine adenovirus (CAV). An in-depth analysis of the epidemiological data indicated that these pathogens actively circulate in the dog populations, and therefore pose a significant threat to susceptible wild carnivore species in the region. The finding that foxes with ongoing or recent CDV infection had a high mortality rate further supported this conclusion. Study findings also suggest that most adult dogs in the population are immune to these pathogens, and play no current or future role in the maintenance or transmission of these pathogens. Unsurprisingly, the village-level vaccination experiment indicated that vaccine-based management of enzootic pathogens in large, free-ranging dog populations would be ineffective and unnecessary, as much of the effort is put into vaccinating dogs that are already antibody positive (thus protected). I used a modeling approach to explore and contrast potential disease control strategies. This work highlights the use of an approach combining data from ecological and epidemiological studies of free-ranging populations with model explorations to better understand disease threats, and identify potential disease management interventions.
\end{abstract}

